\title{
EDITORIALS
}

\section{Beyond Gatekeeping: Enlisting Patients as Agents for Quality and Cost-Containment}

\author{
Richard L. Kravitz \\ Division of General Medicine and Center for Healthcare Policy and Research, University of California, Davis, Sacramento, CA, USA.
}

J Gen Intern Med 23(10):1722-3

DOI: $10.1007 / \mathrm{s} 11606-008-0763-1$

(c) Society of General Internal Medicine 2008

$\mathrm{H}$ ealth care cost inflation is arguably the most fundamental challenge confronting the U.S. health care system. Without effective cost-containment, health insurance premiums will continue to rise, causing employers to drop coverage and putting the price tag for universal insurance further out of reach. ${ }^{1}$ And though quality of care in this country is far from perfect, ${ }^{2}$ most Americans are more worried about affordability than quality. ${ }^{3}$ The causes of rising health care costs are many. However, most health economists finger high utilization of medical technologies (including drugs, devices, and procedures) as the central problem. ${ }^{1,4}$

When judiciously applied, health technologies can deliver substantial clinical value. ${ }^{5}$ However, studies of geographic variation $^{6}$ and of clinical appropriateness ${ }^{7}$ strongly suggest that medical interventions are frequently over-used. This explains how the U.S. manages to spend extravagantly while failing to provide basic health care coverage to 47 million citizens-what Enthoven and Kronick call the "paradox of excess and deprivation." 8 Therefore, the overriding question facing clinicians and health care policymakers over the next several decades is this: how do we ensure the judicious and equitable use of medical technology so as to achieve the greatest possible health benefit at an affordable cost?

While physicians' salaries account for only $20 \%$ of total health care expenditures, doctors control the use of most health care services. Rates of health care spending vary substantially by geographic region in the United States; however, with one exception, increased spending is uncorrelated with improvements in clinical outcome. ${ }^{9}$ That exception is primary care. Multiple ecological studies have shown a positive relationship between primary care supply and improved health outcomes. ${ }^{10}$ Furthermore, since the passage of Medicare in 1965, the one period in which health care inflation approached the rate of general inflation was in the mid-1990s, during the heyday of managed care. The problem with the primary care gatekeeper model isn't that it didn't work. It's that Americans hated it.

The American health care consumer is, like the American health care system itself, a study in paradox. The average (insured) consumer is a product of American history and culture. As such, he or she is drawn inexorably to what Michael Lewis calls the "new new thing," ${ }^{11}$ pulled along by

Published online September 4, 2008 powerful philosophical tides, commercial forces, and a health care system that favors subspecialization over primary care. The surprising thing is that when fully informed about the risks and benefits of interventions, patients tend to exercise far greater discernment, inclining towards more conservative decisions. ${ }^{12}$ Furthermore, Americans want a personal physician and value the relationships they have established with their primary care physicians (PCPs). ${ }^{13}$

The tension created by this paradox is evident in the results of Herndon et al., appearing in this issue of JGIM. ${ }^{14}$ In this well-conceived and executed study, the authors surveyed nearly 3,000 community-dwelling Medicare beneficiaries in 2005 , of whom $82 \%$ had a generalist physician. An almost equal proportion $(79 \%)$ endorsed the statement that "it is better for a patient to have one general doctor who manages most of their problems." Nevertheless, in response to two clinical vignettes (one involving exertional chest pain, the other a persistent cough following an episode of flu), up to threequarters of respondents stated that they would want to have specific diagnostic tests or specialty referrals even if their primary care physician believed them to be unnecessary.

The results suggest that Americans are of two minds when it comes to health care. One mind embraces the continuity, comprehensiveness, and coordination afforded by an ongoing primary care relationship; the other questions whether the PCP's judgment can be fully relied upon. In other words, patients appear to have adopted Ronald Reagan's catchy but oxymoronic dictum to "trust but verify." An important implication is that efforts to rationalize utilization of health care services in the United States will need to address patients not as passive recipients of care but as live actors-replete with hopes, anxieties, desires, and expectations, struggling alongside physicians to do what is best for themselves and their loved ones. This conclusion is consistent with other research showing the substantial influence of patients' expectations and requests on physician behavior. ${ }^{15,16}$ Another implication is that policies designed to strengthen the care coordination role of generalist physicians (e.g., the "advanced medical home") will need to be flexible if they are to avoid backing doctors and their less trusting patients into a corner.

But is this the only reading of the data? Certain aspects of the design cloud the interpretation. Like a Rorschach test, the results are an object of fascination but beg multiple interpretations. As with all surveys that ask about hypothetical circumstances, what patients say they would feel or do is not necessarily what they would actually feel or do during a primary care office visit. Beyond this concern, research suggests that some health care preferences are literally formed in the moment, negotiated in real time as physician and patient exchange information, feelings, and beliefs. ${ }^{17}$ In addi- 
tion, we are told nothing about the length or quality of the patient-PCP relationship; it is reasonable to expect that patients with more secure, enduring, and trustful relationships would be more likely to accede to their physicians' recommendations. Further, the analysis does not control for patients' perceived risk of disease; study participants with a family history of coronary heart disease or a personal history of pneumonia, for example, might be more inclined to press for additional tests or a second opinion. Finally, ordering a noninvasive study on a patient with mild but definite angina is not only reasonable but quite possibly the standard of care (particularly if the test were really without any health risks as described in the vignette-a remarkable situation indeed!). In this particular scenario, patients' reluctance to forego testing suggests not so much moral hazard as good clinical judgment.

Given these ambiguities, varied reactions to this study are predictable. Critics of the medical-industrial complex, already alarmed by capitalist excess dressed as pill-pushing, whole body scanning, and disease mongering, will see in these results evidence for their worst fears: the culture of "more" has infected Grandma and Grandpa. Subspecialty societies, drug companies, and medical device manufacturers will be more sanguine, soothed by the notion that the swell of Boomer retirees can be enlisted as allies in any future battle against rationing or other forms of utilization control. Advocates for consumer-driven health care will claim that elders would play a less diffident tune if held financially accountable for their health care decisions. And generalist physicians, already beleaguered, will take little comfort from authors' conclusion that "If generalists want patients to do less, they will need to do more." Most PCPs will feel that they are doing quite a bit already.

A key question raised by Herndon et al. is how to enlist patients in shared decision-making that is consonant with their values, consistent with the evidence, and respectful of generalist physicians' time and skills. A vision of patients as agents for quality could free patients and doctors from tired stereotypes and frame a creative research agenda. The components of this plan include the following: 1) comprehensive health education beginning in elementary school; 2) interventions throughout the life cycle to support health literacy and numeracy; 3) "Planned Patienthood" programs ${ }^{18}$ that teach people how to use the health care system and leverage the expertise of health professionals-before the medical need arises; 4) a database of patient experiences (perhaps modeled along the lines of PatientsLikeMe.com, only much broader) for use by patients seeking help with tough medical decisions; 5) multimedia tools to support shared decision making; 6) personalized coaching for patients with chronic illness; and 7) computerized decision support and reminder systems to create "trustworthy" practices. Many of these ideas have been tried in isolation, but more comprehensive efforts are needed. Patients as agents for quality would share responsibility for getting the right care, in the right amount, at the right time. Preparing patients for this new role will take both time and resources, but if successful it could reinvigorate primary care, improve outcomes, and help hold the line on costs.

Corresponding Author: Richard L. Kravitz, Division of General Medicine and Center for Healthcare Policy and Research, University of California, Davis, 4150 V. St., Suite 2400 PSSB, Sacramento, CA 95817, USA (e-mail: rlkravitz@ucdavis.edu).

\section{REFERENCES}

1. Emanuel EJ, Fuchs VR. The perfect storm of overutilization. Jama. 2008;299(23):2789-91, Jun 18

2. Committee on Quality of Health Care in America of the Institute of Medicine. Crossing the Quality Chasm: A New Health System for the 21st Century. Washington, D.C.: National Academy Press; 2001.

3. Blendon RJ, Brodie M, Benson JM, Altman DE, Buhr T. Americans' views of health care costs, access, and quality. Milbank Q. 2006;84 (4):623-57.

4. Bodenheimer T. High and rising health care costs. Part 2: technologic innovation. Ann Intern Med. 2005;142(11):932-7, Jun 7.

5. Cutler DM, McClellan M. Is technological change in medicine worth it? Health Aff (Millwood). 2001;20(5):11-29, Sep-Oct

6. Wennberg J, Fisher E, Goodman D, Skinner J. Tracking the Care of Patients with Severe Chronic Illness: The Dartmouth Atlas of Health Care 2008. Lebanon, New Hampshire: Dartmouth Institute for Health Policy and Clinical Practice, Center for Health Policy Research; 2008.

7. Leape L. Unnecessary surgery. Annu Rev Public Health. 1989;13:36383.

8. Enthoven A, Kronick R. A consumer-choice health plan for the 1990s. Universal health insurance in a system designed to promote quality and economy (1). N Engl J Med. 1989;320(1):29-37, Jan 5.

9. Fisher E, Wennberg D, Stukel T, Gottlieb D, Lucas F, Pinder E. The implications of regional variations in Medicare spending. Part 2: health outcomes and satisfaction with care. Ann Intern Med. 2003;138(4):28898.

10. Macinko J, Starfield B, Shi L. Quantifying the health benefits of primary care physician supply in the United States. Int J Health Serv. 2007;37(1): 111-26.

11. Lewis MM. The New New Thing: A Silicon Valley Story. New York: WW Norton; 2000

12. O'Connor A, Llewellyn-Thomas H, Flood A. Modifying unwarranted variations in health care: shared decision making using patient decision aids. Health Aff (Millwood). 2004;Suppl Web Exclusives:VAR63-72.

13. Safran D. Defining the future of primary care: what can we learn from patients? Ann Intern Med. 2003; 138(3):248-55.

14. Herndon B, Schwartz LM, Woloshin S, Anthony D, Gallagher P, Fowler FJ, Fisher E. Older patients perceptions of "unnecessary" tests and referrals. J Gen Intern Med. October, 2008; xxx-xxx.

15. Keitz SA, Stechuchak KM, Grambow SC, Koropchak CM, Tulsky JA. Behind closed doors: management of patient expectations in primary care practices. Arch Intern Med. 2007;167(5):445-52, Mar 12.

16. Kravitz RL. Patients' expectations for medical care: an expanded formulation based on review of the literature. Med Care Res Rev. 1996;53(1):3-27, Mar.

17. Peters E, Dieckmann N. Less is more in presenting quality information to consumers. Med Care Res Rev. 2007;64:169-90.

18. Kaplan S. Interpreting Controversial Diabetes Trials for Patients and their Doctors. Sacramento, CA: UC Davis Internal Medicine Grand Rounds; 2008. 